QUARTERLY OF APPLIED MATHEMATICS

VOLUME LXIII, NUMBER 2

JUNE 2005, PAGES 201-223

S $0033-569 \mathrm{X}(05) 00952-6$

Article electronically published on February 18, 2005

\title{
LOCALIZED SOLUTIONS OF A NONLINEAR DIATOMIC LATTICE
}

\author{
BY \\ V. V. KONOTOP (Centro de Física Teórica e Computacional, Universidade de Lisboa, Complexo \\ Interdisciplinar, Av. Professor Gama Pinto 2, 1649-003 Lisbon, Portugal) \\ AND \\ G. PERLA MENZALA (National Laboratory of Scientific Computation, LNCC/MCT, Rua Getulio \\ Vargas 333, Petropolis, RJ, CEP 25651-070, Brasil and Federal University of Rio de Janeiro, \\ Institute of Mathematics, P.O. Box 68530, 21945-970, Rio de Janeiro, RJ, Brasil)
}

\begin{abstract}
We consider a coupled system of differential-difference nonlinear equations. We study the dynamics of such a diatomic lattice showing global existence and uniqueness in an appropriate function space. Our approach based on energy estimates allows us to prove the result only in the case where nonlinear force constants are positive and equal. All other situations remain at this point as open problems.
\end{abstract}

1. Introduction. Nonlinear lattices, i.e. systems of coupled differential-difference nonlinear equations, constitute a subject attracting nowdays a great deal of attention in physical literature [6]. They describe numerous physical phenomena, like, for example, the conventional atomic lattice making up solids [8] and macromolecules [6]. As nonlinear lattices (either finite or infinite) are in general not integrable, i.e. neither possess an infinite number of integrals of motion nor allow an explicit solution of the respective Cauchy problem, the main attention in the physical literature has been devoted either to searching for particular solutions with a subsequent study of their stability or to developing various approximations. As an example we mention the so-called multiple scale expansion (see e.g. 3] and references therein). Being rather effective in finding particular approximate solutions, this method remains reliable as an analogy of the discrete system to a continuous subject to definite conditions and naturally does not allow one to make any conclusion about the global long-time dynamics of the lattice. In particular, if one considers a lattice with a quadratic or cubic nonlinearity, then the resulting approximate evolution equation is either the Korteweg-de Vries (KdV) equation (if neighbor atoms experience in-phase oscillations, i.e. the respective wave-vector is in the center of the Brillouin zone) or the nonlinear Schrödinger (NLS) equation (if neighbor atoms experience

Received March, 2003.

2000 Mathematics Subject Classification. Primary 39A10, 39B72.

Key words and phrases. Nonlinear lattices, high interaction, diatomic case.

E-mail address: konotop@cii.fc.ul.pt

E-mail address: perla@lncc.br

(C)2005 Brown University 
out-of-phase oscillations, i.e. the respective wave-vector is located at the boundary of the Brillouin zone). If the underlying lattice has nonlinearity higher than the third one, then the resulting equation is either a modified $\mathrm{KdV}$ equation with higher nonlinearity or it is an NLS equation with higher nonlinearity. This last fact becomes especially important because on the one hand each of the above partial differential equations can display a blow-up (or collapse) phenomenon (see e.g. [2] and 7], respectively) and on the other hand the long-time behavior cannot be investigated by any of the known approximate methods and at the same time, the blow-up should be prevented by the discreteness, which defines the smallest spatial scale of the problem equal to the distance between neighbor atoms. Thus, the well-known results for nonlinear partial differential equations are not applicable for the lattices. Another feature of lattice equations to be mentioned here is the ambiguity of the continuum limit, which is achieved by different choices of the scaling. Moreover, in certain cases a lattice possesses properties having no analogy in the continuum limit. This is, in particular, the case of a diatomic lattice, which in the linear limit is characterized by two branches of the spectrum of linear excitations. Finally, even in the case of approximate equations which do not display blow-up, the phenomenon of modulational instability can exist [1. This phenomenon has as a consequence possible growth of the solution locally in time. Such a growth, for example, makes the conditions of the applicability of multiple-scale expansion (usually it is the requirement of smallness of the amplitude of the excitation) not valid anymore and thus leaves open the problem of long-time evolution of the lattice excitations.

The situation described above has stimulated our previous studies where we have proven the global existence of solutions of the highly nonlinear infinite Fermi-PastaUlam lattice [4] and the long-time asymptotic of the solutions of a finite lattice with dissipation subject to periodic boundary conditions [5]. The respective proofs, however, have been presented for the case of a monoatomic lattice. Due to more than one branch of the spectrum, a bi- or multi-atomic lattice (i.e. a lattice with a complex cell) possesses a number of peculiarities compared with its monoatomic counterpart. One relevant to the subject of the present paper is the instability of the solutions. Indeed, for the same type of the nonlinearity and the same amplitude, a stable solution at one frequency may become unstable at another frequency.

The aim of the present work is to extend the proof presented in [4] to the case of a diatomic one-dimensional lattice. More specifically, in Section 2 we formulate the Cauchy problem for the diatomic lattices in terms of the relative displacements and list the main properties of the associated Green function. In Section 3 we introduce the energy space and prove local existence of the solution in that function space. Global existence in proven in Section 4. We summarize the results and mention some open problem in Section 5.

2. Statement of the Problem and Preliminaries. The main purpose of this work is to prove a global existence (and uniqueness) of a solution of a nonlinear diatomic 
lattice which is described by the Hamiltonian

$$
\begin{aligned}
H & =\frac{M_{1}}{2} \sum_{n=-\infty}^{+\infty}\left[\dot{u}_{1}(n, t)\right]^{2}+\frac{M_{2}}{2} \sum_{n=-\infty}^{+\infty}\left[\dot{u}_{2}(n, t)\right]^{2} \\
& +\frac{K_{1}}{2} \sum_{n=-\infty}^{+\infty}\left[u_{2}(n, t)-u_{1}(n, t)\right]^{2}+\frac{K_{2}}{2} \sum_{n=-\infty}^{+\infty}\left[u_{1}(n, t)-u_{2}(n-1, t)\right]^{2} \\
& +\frac{V_{1}}{p+1} \sum_{n=-\infty}^{+\infty}\left[u_{2}(n, t)-u_{1}(n, t)\right]^{p+1}+\frac{V_{2}}{p+1} \sum_{n=-\infty}^{+\infty}\left[u_{1}(n, t)-u_{2}(n-1, t)\right]^{p+1} .
\end{aligned}
$$

In (2.1), $u_{j}(n, t)(j=1,2)$ denotes the displacement of the $j$-th atom located in $n$-th cell and having the mass $M_{j}\left(M_{j}>0\right)$. The positive constants $K_{j}$ and $V_{j}$ are associated to linear and nonlinear force constants, respectively. Finally, $p$ is an odd integer, $p \geq 3$, and a dot stands for the derivative with respect to $t$.

The evolution equations for the lattice displacements read as follows:

$$
\begin{aligned}
M_{1} \ddot{u}_{1}(n, t)= & -K_{1}\left[u_{1}(n, t)-u_{2}(n, t)\right]-K_{2}\left[u_{1}(n, t)-u_{2}(n-1, t)\right] \\
& -V_{1}\left[u_{1}(n, t)-u_{2}(n, t)\right]^{p}-V_{2}\left[u_{1}(n, t)-u_{2}(n-1, t)\right]^{p}, \\
M_{2} \ddot{u}_{2}(n, t)= & -K_{1}\left[u_{2}(n, t)-u_{1}(n, t)\right]-K_{2}\left[u_{2}(n, t)-u_{1}(n+1, t)\right], \\
& -V_{1}\left[u_{2}(n, t)-u_{1}(n, t)\right]^{p}-V_{2}\left[u_{2}(n, t)-u_{1}(n+1, t)\right]^{p} .
\end{aligned}
$$

We will require some simplifications in (2.2), (2.3) before we begin our discussion. Multiply equations (2.2) and (2.3) by $1 / M_{1}$ and $1 / M_{2}$, respectively. Subtracting the resulting corresponding equations and introducing the relative displacements

$$
\alpha(n, t)=u_{2}(n, t)-u_{1}(n, t), \quad \alpha_{2}(n, t)=u_{1}(n, t)-u_{2}(n-1, t),
$$

one can verify that $\alpha_{1}$ and $\alpha_{2}$ do satisfy the coupled system

$$
\begin{aligned}
\ddot{\alpha}_{1}(n, t)= & -\frac{K_{1}}{M} \alpha_{1}(n, t)+\frac{K_{2}}{M_{2}} \alpha_{2}(n+1, t)+\frac{K_{2}}{M_{1}} \alpha_{2}(n, t) \\
& -\frac{V_{1}}{M}\left[\alpha_{1}(n, t)\right]^{p}+\frac{V_{2}}{M_{2}}\left[\alpha_{2}(n+1, t)\right]^{p}+\frac{V_{2}}{M_{1}}\left[\alpha_{2}(n, t)\right]^{p}, \\
\ddot{\alpha}_{2}(n, t)= & -\frac{K_{2}}{M} \alpha_{2}(n, t)+\frac{K_{1}}{M_{1}} \alpha_{2}(n, t)+\frac{K_{1}}{M_{2}} \alpha_{1}(n-1, t) \\
& -\frac{V_{2}}{M}\left[\alpha_{2}(n, t)\right]^{p}+\frac{V_{1}}{M_{1}}\left[\alpha_{1}(n+1, t)\right]^{p}+\frac{V_{1}}{M_{2}}\left[\alpha_{1}(n-1, t)\right]^{p}
\end{aligned}
$$

where $M=\frac{M_{1} M_{2}}{M_{1}+M_{2}}$. Considering a new time variable $\tilde{t}=\left(K_{1}^{1 / 4} K_{2}^{1 / 4} / \sqrt{M}\right) t$ and denoting the constants $\kappa, m_{j}$, and $\gamma_{j}(j=1,2)$ by

$$
m_{j}=\frac{M}{M_{j}}, \quad \kappa=\sqrt{\frac{K_{1}}{K_{2}}}, \quad \gamma_{j}=\frac{V_{j}}{\sqrt{K_{1} K_{2}}},
$$


we can rewrite (2.5) and (2.6) as

$$
\begin{aligned}
\ddot{\alpha}_{1}(n, \tilde{t})= & -\kappa \alpha_{1}(n, \tilde{t})+\frac{m_{2}}{\kappa} \alpha_{2}(n+1, \tilde{t})+\frac{m_{1}}{\kappa} \alpha_{2}(n, \tilde{t}) \\
& -\gamma_{1}\left[\alpha_{1}(n, \tilde{t})\right]^{p}+\gamma_{2} m_{2}\left[\alpha_{2}(n+1, \tilde{t})\right]^{p}+\gamma_{2} m_{1}\left[\alpha_{2}(n, \tilde{t})\right]^{p}, \\
\ddot{\alpha}_{2}(n, \tilde{t})= & -\frac{1}{\kappa} \alpha_{2}(n, \tilde{t})+m_{1} \kappa \alpha_{1}(n, \tilde{t})+m_{2} \kappa \alpha_{1}(n-1, \tilde{t}) \\
& -\gamma_{2}\left[\alpha_{1}(n, \tilde{t})\right]^{p}+\gamma_{1} m_{1}\left[\alpha_{1}(n, \tilde{t})\right]^{p}+\gamma_{1} m_{2}\left[\alpha_{1}(n-1, \tilde{t})\right]^{p} .
\end{aligned}
$$

Hereafter we drop the tilde over the temporal variable, since we will deal only with the evolution equations in the form (2.7), (2.8).

Let us analyze the linear part of the system (2.7) and (2.8), that is, the pair $\left\{\beta_{1}(n, t)\right.$, $\left.\beta_{2}(n, t)\right\}$, solution of the problem

$$
\left\{\begin{array}{l}
\ddot{\beta}_{1}(n, t)=-\kappa \beta_{1}(n, t)+m_{2} \kappa^{-1} \beta_{2}(n+1, t)+m_{1} \kappa^{-1} \beta_{2}(n, t), \\
\ddot{\beta}_{2}(n, t)=-\kappa^{-1} \beta_{2}(n, t)+\kappa m_{1} \beta_{1}(n, t)+\kappa m_{2} \beta_{1}(n-1, t), \\
\beta_{j}(n, 0)=\tilde{\alpha}_{j}(n), \quad \dot{\beta}_{j}(n, 0)=\tilde{b}_{j}(n) \quad(j=1,2) \\
\lim _{n \rightarrow \pm \infty} \beta_{j}(n, t)=0 \quad(j=1,2)
\end{array}\right.
$$

where $\tilde{\alpha}_{j}=\alpha_{j}(n, 0)$ and $\tilde{b}_{j}=\dot{\alpha}_{j}(n, 0)$. The two-component Green function $G(n, t)=$ $\left\{G_{1}(n, t), G_{2}(n, t)\right\}$ associated with (2.9) can be explicity calculated and each component is given by

$$
G_{j}(n, t)=\frac{1}{\pi} \int_{0}^{\pi / 2} H_{j}(n, t, \sigma) d \sigma
$$

where

$$
\begin{aligned}
H_{j}(n, t, \sigma) & =\frac{\kappa^{2(j-1)}}{\left(1+\kappa^{2}\right) \Delta(\sigma)}\left[\left(1+m_{1}\right) \cos (2 \sigma n)+m_{2} \cos \left(2 \sigma\left(n-(-1)^{j}\right)\right)\right] \\
& \times\left(\frac{\sin \left(\omega_{-} t\right)}{\omega_{-}}-\frac{\sin \left(\omega_{+} t\right)}{\omega_{+}}\right)+\cos (2 \sigma n)\left(\frac{\sin \left(\omega_{-} t\right)}{\omega_{-}}+\frac{\sin \left(\omega_{+} t\right)}{\omega_{+}}\right) .
\end{aligned}
$$

Here

$$
\Delta(\sigma)=\sqrt{1-16 \frac{m_{1} m_{2}}{\left(\kappa+\kappa^{-1}\right)^{2}} \sin ^{2}(\sigma)}
$$

and

$$
\omega_{ \pm}^{2} \equiv \omega_{ \pm}^{2}(\sigma)=\left(\kappa+\kappa^{-1}\right)(1 \pm \Delta(\sigma))
$$

is the dispersion relation of the diatomic lattice.

Clearly, the above representation for $H_{j}$ requires that $\Delta(\sigma)>0$ for all $0 \leq \sigma \leq \pi / 2$. Later on, we will need, due to technical matters, that $\kappa=1$ (which in physical terms means equality of the linear force constants). Therefore, from now on we will assume the following condition.

Condition 1. $\left(m_{1}, m_{2}\right)$ is different from $\left(\frac{1}{2}, \frac{1}{2}\right)$ and $\kappa=1$.

Thus, Condition 1 in particular implies that

$$
\Delta_{\min }=\min _{0 \leq \sigma \leq \pi / 2} \Delta(\sigma)>0 .
$$


The components of the Green function can be bounded as follows $(j=1,2)$ :

$$
\begin{aligned}
\left|G_{j}(0, t)\right| & \leq C|t|, \\
\left|G_{j}\left((-1)^{j}, t\right)\right| & \leq C|t|, \\
\left|G_{j}(n, t)\right| & \leq C \frac{|t|+|t|^{3}}{n^{2}}, \quad \forall n \neq 0,(-1)^{j} .
\end{aligned}
$$

Hereafter various positive constants will be denoted as $C$, but we remark that they may vary from line to line.

Inequalities (2.14), (2.15) are trivial. Let us briefly indicate the proof of (2.16) for $G_{1}(n, t)$ (the proof for $G_{2}(n, t)$ is similar). We write $G_{1}(n, t)$ as

$$
G_{1}(n, t)=\frac{1}{\pi} \sum_{j=1}^{4} \int_{0}^{\pi / 2} J_{j}(n, t, \sigma) d \sigma
$$

where

$$
\begin{aligned}
& J_{1}=\left[f_{1}(\sigma) \cos (2 \sigma n)+f_{2}(\sigma) \cos (2 \sigma(n+1))\right] \frac{\sin \left(\omega_{-} t\right)}{\omega_{-}} \\
& J_{2}=-\left[f_{1}(\sigma) \cos (2 \sigma n)+f_{2}(\sigma) \cos (2 \sigma(n+1))\right] \frac{\sin \left(\omega_{+} t\right)}{\omega_{+}} \\
& J_{3}=\cos (2 \sigma n) \frac{\sin \left(\omega_{-} t\right)}{\omega_{-}} \\
& J_{4}=\cos (2 \sigma n) \frac{\sin \left(\omega_{+} t\right)}{\omega_{+}}
\end{aligned}
$$

and

$$
f_{1}=\frac{1+m_{1}}{2 \Delta(\sigma)}, \quad f_{2}=\frac{m_{2}}{2 \Delta(\sigma)}
$$

The analysis of all terms will be very similar; therefore we will illustrate the steps of just one of them, for instance

$$
I^{0}(n, t)=\frac{1}{\pi} \int_{0}^{\pi / 2} f_{1}(\sigma) \cos (2 \sigma n) \frac{\sin \left(\omega_{-} t\right)}{\omega_{-}} d \sigma .
$$

Since $\omega_{-}(0)=0$, we consider

$$
I^{\varepsilon}=I^{\varepsilon}(n, t)=\frac{1}{\pi} \int_{\varepsilon}^{\pi / 2} f_{1}(\sigma) \cos (2 \sigma n) \frac{\sin \left(\omega_{-} t\right)}{\omega_{-}} d \sigma
$$

with $\varepsilon>0$. Integration by parts twice gives

$$
I^{\varepsilon}=I_{1}^{\varepsilon}+I_{2}^{\varepsilon}-\frac{1}{4 \pi n^{2}} \int_{\varepsilon}^{\pi / 2} \cos (2 \sigma n) \frac{\partial^{2}}{\partial \sigma^{2}}\left\{f_{1}(\sigma) \frac{\sin \left(\omega_{-} t\right)}{\omega_{-}}\right\} d \sigma
$$

where

$$
\begin{aligned}
& I_{1}^{\varepsilon}=-\frac{1}{2 \pi n} \sin (2 \varepsilon n) f_{1}(\varepsilon) \frac{\sin \left(\omega_{-}(\varepsilon) t\right)}{\omega_{-}(\varepsilon)} \\
& I_{2}^{\varepsilon}=\frac{1}{2 \pi n}\left[\frac{\cos (2 \varepsilon n)}{2 n} \frac{\partial}{\partial \sigma}\left\{f_{1}(\varepsilon) \frac{\sin \left(\omega_{-}(\varepsilon) t\right)}{\omega_{-}(\varepsilon)}\right\}\right]_{t=\varepsilon}^{t=\pi / 2}
\end{aligned}
$$


Since $\lim _{\varepsilon \rightarrow 0} I_{1}^{\varepsilon}=\lim _{\varepsilon \rightarrow 0} I_{2}^{\varepsilon}=0$, we obtain as $\varepsilon \rightarrow 0$ that

$$
\lim _{\varepsilon \rightarrow 0} I^{\varepsilon}=I^{0}=-\frac{1}{4 \pi n^{2}} \int_{0}^{\pi / 2} \cos (2 \sigma n) \frac{\partial^{2}}{\partial \sigma^{2}}\left\{f_{1}(\sigma) \frac{\sin \left(\omega_{-} t\right)}{\omega_{-}}\right\} d \sigma .
$$

Direct calculations show that

$$
\frac{\partial^{2}}{\partial \sigma^{2}}\left\{f_{1}(\sigma) \frac{\sin \left(\omega_{-} t\right)}{\omega_{-}}\right\}=f_{1}^{(2)} \chi_{1}+f_{1}^{(1)} \chi_{2}+f_{1} \chi_{3}
$$

where

$$
\begin{aligned}
\chi_{1} & =\omega_{-}^{-1} \sin \left(\omega_{-} t\right), \\
\chi_{2} & =\omega_{-}^{-2}\left[\omega_{-} \omega_{-}^{(1)} t \cos \left(\omega_{-} t\right)-\omega_{-}^{(1)} \sin \left(\omega_{-} t\right)\right], \\
\chi_{3} & =\omega_{-}^{-2}\left[-\omega_{-}\left(\omega_{-}^{(1)}\right)^{2} t^{2} \sin \left(\omega_{-} t\right)+t \cos \left(\omega_{-} t\right)\left\{\left(\omega_{-}^{(1)}\right)^{2}+\omega_{-} \omega_{-}^{(2)}\right\}\right. \\
& \left.-\omega_{-}^{(2)} \sin \left(\omega_{-} t\right)-t\left(\omega_{-}^{(1)}\right)^{2} \cos \left(\omega_{-} t\right)\right]-2 \omega_{-}^{-3} \omega_{-}^{(1)}\left[\omega_{-} \omega_{-}^{(1)} \cos \left(\omega_{-} t\right)-\omega_{-}^{(1)} \sin \left(\omega_{-} t\right)\right] .
\end{aligned}
$$

Here

$$
\omega_{-}^{(m)}=\frac{d^{m} \omega_{-}}{d \sigma^{m}} \quad \text { and } \quad f_{1}^{(1)}=\frac{d^{m} f_{1}}{d \sigma^{m}}, \quad m=1,2 .
$$

We claim that
(a) $\left|f_{1}^{(2)} \chi_{1}\right| \leq C|t|$,
(b) $\left|f_{1}^{(1)} \chi_{2}\right| \leq C|t|^{3}$,
(c) $\left|f_{1}^{(1)} \chi_{3}\right| \leq C|t|^{3}$

for all $t>0$ and some positive constant $C$. In fact, (a) follows because the estimate $\left|f_{1}^{(2)}\right| \leq C \Delta_{\text {min }}^{-1}$ holds where $\Delta_{\text {min }}$ is given by (2.13). To prove (b), we consider

$$
|X|=t^{2}\left|\frac{\omega_{-} t \cos \left(\omega_{-} t\right)-\sin \left(\omega_{-} t\right)}{t^{2} \omega_{-}^{2}}\right|
$$

for $t>0(\sigma>0)$, and denoting $Y=\omega_{-}(\sigma) t$, we have that

$$
X=t^{2}\left[\frac{Y-\sin Y}{Y^{2}}-\frac{2 \sin ^{2}(Y / 2)}{Y}\right] \leq t^{2}\left[\left|\frac{Y-\sin Y}{Y^{2}}\right|+\frac{|Y|}{2}\right] \leq \frac{7}{6}|Y| t^{2}
$$

(because $|Y-\sin Y| \leq|Y|^{3} / 6$ for $t>0,0<\sigma<\pi / 2$. Thus, we conclude that

$$
\left|f_{1}^{(1)} \chi_{2}\right| \leq C\left|f_{1}^{(1)}\right|\left|\omega_{-}^{(1)}\right||t|^{2}|Y| \leq C\left|f_{1}^{(1)}\right|\left|\omega_{-}^{(1)}\right|\left|\omega_{-}\right||t|^{3}
$$

which proves (b) because $f_{1}^{(1)}, \omega_{-}^{(1)}$, and $\omega_{-}$are bounded by a positive constant. A similar discussion proves (c), so we will omit it. Using (a), (b), and (c) together with (2.18), it follows that we obtained a bound for $I^{0}(n, t)$ of type $c \frac{|t|+|t|^{2}}{n^{2}} \cdot$ All other terms in $J_{i} \quad(i=1,2,3,4)$ are treated similarly.

We will also need to consider the derivatives of the Green function which are obtained from $(2.10)(j=1,2)$ :

$$
\dot{G}_{j}(n, t)=\frac{1}{\pi} \int_{0}^{\pi / 2} \dot{H}_{j}(n, t, \sigma) d \sigma
$$


together with their estimates similar to (2.14)-(2.16) for $G_{j}$ :

$$
\begin{aligned}
\left|\dot{G}_{j}(0, t)\right| & \leq C t^{2}, \\
\left|\dot{G}_{j}\left((-1)^{j}, t\right)\right| & \leq C t^{2}, \\
\left|\dot{G}_{j}(n, t)\right| & \leq C\left(\frac{t^{2}+t^{4}}{|n|^{3}}+\frac{t^{4}}{\left|n-(-1)^{j}\right|^{3}}\right), \quad \forall n \neq 0,(-1)^{j} .
\end{aligned}
$$

Again, (2.20) and (2.21) are trivial and we can prove (2.22) following the steps described above.

3. Existence of a Local Solution. Let $T>0$. We consider the linear space $H=$ $H(T)$ consisting of all functions $\alpha(t)$ of the form:

$$
\alpha(t)=\left(\cdots ; \alpha_{1}(j-1, t), \alpha_{2}(j-1, t), \alpha_{1}(j, t), \alpha_{2}(j, t) ; \alpha_{1}(j+1, t), \alpha_{2}(j+1, t) \cdots\right)
$$

with $\alpha_{m}: \mathbb{Z} \times[0, T) \mapsto \mathbb{R}$ such that

(a) each $\alpha_{m}(n, t)$ is in class $C^{2}$ in the variable $t$,

$$
\begin{aligned}
\text { (b) } \sup _{0 \leq t<T}\left\{\sum _ { n = - \infty } ^ { + \infty } \left[\frac{1}{m_{1}}\left(\sum_{\ell=-\infty}^{n}\left[\dot{\alpha}_{2}(\ell, t)+\dot{\alpha}_{1}(\ell-1, t)\right]\right)^{2}\right.\right. \\
\left.\left.+\frac{1}{m_{2}}\left(\sum_{\ell=-\infty}^{n}\left[\dot{\alpha}_{2}(\ell, t)+\dot{\alpha}_{1}(\ell-1, t)\right]\right)^{2}\right]+\sum_{n=-\infty}^{+\infty}\left[\alpha_{1}^{2}(n, t)+\alpha_{2}^{2}(n, 1)\right]\right\}<+\infty
\end{aligned}
$$

where the positive constants $m_{1}$ and $m_{2}$ are as in Section 2 (we are assuming Condition 1 ) and $\mathbb{Z}$ denotes the set of integers. The space $H=H(T)$ will be referred to as the energy space. We define the norm in $H$ as

$$
\begin{aligned}
\|\alpha(\cdot)\|_{H}^{2} & =\sup _{0 \leq t<T}\left\{\frac { 1 } { 2 } \sum _ { n = - \infty } ^ { + \infty } \left[\frac{1}{m_{1}}\left(\sum_{\ell=-\infty}^{n}\left[\dot{\alpha}_{2}(\ell, t)+\dot{\alpha}_{1}(\ell-1, t)\right]\right)^{2}\right.\right. \\
& \left.\left.+\frac{1}{m_{2}}\left(\sum_{\ell=-\infty}^{n}\left[\dot{\alpha}_{2}(\ell, t)+\dot{\alpha}_{1}(\ell, t)\right]\right)^{2}\right]+\frac{1}{2} \sum_{n=-\infty}^{+\infty}\left[\alpha_{1}^{2}(n, t)+\alpha_{2}^{2}(n, t)\right]\right\} .
\end{aligned}
$$

Clearly $\left(H,\|\cdot\|_{H}\right)$ becomes a Banach space.

Let $\left(\beta_{1}, \beta_{2}\right)$ be the solution of $(2.9)$ where $\left\{\tilde{a}_{j}(n)\right\}$ and $\left\{\tilde{b}_{j}(n)\right\}$ satisfy

$$
\begin{aligned}
\frac{1}{2 m_{1}} \sum_{n=-\infty}^{+\infty}\left(\sum_{\ell=-\infty}^{n}\left[\tilde{b}_{2}(\ell)+\tilde{b}_{1}(\ell-1)\right]\right)^{2} & +\frac{1}{2 m_{2}} \sum_{n=-\infty}^{+\infty}\left(\sum_{\ell=-\infty}^{n}\left[\tilde{b}_{2}(\ell)+\tilde{b}_{1}(\ell)\right]\right)^{2} \\
& +\frac{1}{2} \sum_{n=-\infty}^{+\infty}\left[\left|\tilde{a}_{1}(n)\right|^{2}+\left|\tilde{a}_{2}(n)\right|^{2}\right]<\infty
\end{aligned}
$$

LEMMA 3.1. Let

$$
\beta(t)=\left(\cdots ; \beta_{1}(j-1, t), \beta_{2}(j-1, t) ; \beta_{1}(j, t), \beta_{2}(j, t) ; \beta_{1}(j+1, t), \beta_{2}(j+1, t) ; \cdots\right)
$$


where the pair $\left(\beta_{1}(n, t), \beta_{2}(n, t)\right)$ is the solution of problem $(2.9)$ with $\left\{\tilde{a}_{j}(n)\right\}$ and $\left\{\tilde{b}_{j}(n)\right\}$ satisfying (3.2). Then, for any $T>0$, the function $\beta(t)(0 \leq t<T)$ belongs to the energy space $H(T)$.

Proof. The result follows using the explicit form of the solution of (2.9) given by

$$
\beta_{j}(n, t)=\sum_{m=-\infty}^{+\infty}\left[G_{j}(n-m, t) \tilde{b}_{j}(m)+\dot{G}_{j}(n-m, t) \tilde{a}_{j}(m)\right]
$$

and the properties of the Green function $\left(G_{1}(n, t), G_{2}(n, t)\right)$.

We rewrite the evolution equations $(2.7),(2.8)$ in the integral from

$$
\begin{array}{r}
\alpha_{1}(n, t)=\beta_{1}(n, t)+\sum_{\ell=-\infty}^{+\infty} \int_{0}^{t} G_{1}(n-\ell, t-s)\left\{-\gamma_{1}\left[\alpha_{1}(\ell, s)\right]^{p}\right. \\
\left.+\gamma_{2} m_{2}\left[\alpha_{2}(\ell+1, s)\right]^{p}+\gamma_{2} m_{1}[\alpha(\ell, s)]^{p}\right\} d s, \\
\begin{aligned}
\alpha_{2}(n, t)=\beta_{2}(n, t)+ & \sum_{\ell=-\infty}^{+\infty} \int_{0}^{t} G_{2}(n-\ell, t-s)\left\{-\gamma_{2}\left[\alpha_{2}(\ell, s)\right]^{p}\right. \\
& \left.+\gamma_{1} m_{1}\left[\alpha_{1}(\ell, s)\right]^{p}+\gamma_{1} m_{2}\left[\alpha_{2}(\ell-1, s)\right]^{p}\right\} d s
\end{aligned}
\end{array}
$$

where $\beta_{j}(n, t)$ is a solution of problem (2.9).

From now on we will also assume

Condition 2. $\gamma_{1}=\gamma_{2}=\gamma$ (i.e. the lattice with equal nonlinear interactions).

Theorem 3.1 (Local existence). Let us assume that Conditions 1 and 2 are valid with $\left\{\tilde{a}_{n}\right\}$ and $\left\{\tilde{b}_{2}\right\}$ satisfying (3.2). Furthermore, assume that $p$ is an odd integer $\geq 3$. Then, there exists $T>0$ and a unique function $\alpha(t) \in H(T)$,

$$
\alpha(t)=\left(\cdots ; \alpha_{1}(j-1, t), \alpha_{2}(j-1, t) ; \alpha_{1}(j, t), \alpha_{2}(j, t) ; \alpha_{1}(j+1, t), \alpha_{2}(j+1, t) ; \cdots\right),
$$

defined in $[0, T)$, such that $\left\{\alpha_{1}(n, t), \alpha_{2}(n, t)\right\}_{n=-\infty}^{+\infty}$ solves $(2.7),(2.8)$ with initial data $\left\{\tilde{a}_{j}(n)\right\}$ and $\left\{\tilde{b}_{j}(n)\right\}$.

Proof. Let $R>0$ and $T>0$. We define the subset

$$
\begin{aligned}
F_{R}=\left\{\alpha(t) \in H,\|\alpha(\cdot)-\beta(\cdot)\|_{H} \leq R ; \alpha_{j}(n, 0)=\beta_{j}(n, 0), \dot{\alpha}_{j}(n, 0)\right. & =\dot{\beta}_{j}(n, 0), \\
j & =1,2 \forall n \in \mathbb{Z}\} .
\end{aligned}
$$

Clearly, $F_{R}$ is a closed subset of $H$. For any element $\alpha(t) \in F_{R}(T)$, we define the map $P_{\alpha}(t)$ given by

$$
P \alpha(t)=\left(\cdots ; \widetilde{P}_{1} \alpha_{1}(j-1, t), \widetilde{P}_{2} \alpha_{2}(j-1, t) ; \widetilde{P}_{1} \alpha_{1}(j, t), \widetilde{P}_{2} \alpha_{2}(j, t) ; \cdots\right)
$$

where

$$
\widetilde{P}_{j} \alpha_{j}(n, t)=\beta_{j}(n, t)+\gamma \sum_{\ell=-\infty}^{+\infty} \int_{0}^{t} G_{j}(n-\ell, t-s) Q_{j}\left(\alpha_{1}, \alpha_{2}\right)(\ell, s) d s, \quad j=1,2,
$$

with

$$
\begin{aligned}
& Q_{1}\left(\alpha_{1}, \alpha_{2}\right)(\ell, s)=m_{2} \alpha_{2}^{p}(\ell+1, s)+m_{1} \alpha_{2}^{p}(\ell, s)-\alpha_{1}^{p}(\ell, s), \\
& Q_{2}\left(\alpha_{1}, \alpha_{2}\right)(\ell, s)=m_{2} \alpha_{1}^{p}(\ell-1, s)+m_{1} \alpha_{1}^{p}(\ell, s)-\alpha_{2}^{p}(\ell, s) .
\end{aligned}
$$


Observe that

$$
\widetilde{P}_{j} \alpha_{j}(n, t=0)=\tilde{a}_{j}(n), \quad \frac{d}{d t} \widetilde{P}_{j} \alpha_{j}(n, t=0)=\tilde{b}_{j}(n), \quad j=1,2 .
$$

We will prove that the map $P$ sends the elements of the closed subset $F_{R}(T) \subseteq H(T)$ into $F_{R}$ and it is a contraction there, that is, there exists $0<\delta<1$ such that

$$
\|P f-P g\|_{H} \leq \delta\|f-g\|_{H}, \quad \forall f, g \in F_{R}
$$

provided $T>0$ is chosen sufficiently small.

Due to Lemma 3.1, in order to prove that for a given $\alpha \in H, P_{\alpha}$ belongs to $H(T)$, it is sufficient to show that $P_{\alpha}-\beta$ belongs to $H$. Let $\alpha \in H(T)$ and denote by $w_{j}(n, t)$ the function

$$
w_{j}(n, t)=\widetilde{P}_{j} \alpha_{j}(n, t)-\beta_{j}(n, t)=\gamma \sum_{\ell=-\infty}^{+\infty} \int_{0}^{t} G_{j}(n-\ell, t-s) Q_{j}\left(\alpha_{2}, \alpha_{2}\right)(\ell, s) d s
$$

for $j=1,2$. We need to prove that the map

$$
w(t)=\left(\cdots ; w_{1}(j-1, t), w_{2}(j-1, t) ; w_{1}(j, t), w_{2}(j, t) ; \cdots\right)
$$

belongs to $H(T)$, that is,

$$
\sup _{0 \leq t<T} \sum_{n=-\infty}^{+\infty}\left\{\sum_{\ell=-\infty}^{n}\left[\dot{w}_{1}(\ell, t)+\dot{w}_{1}(\ell-1, t)\right]\right\}^{2}<+\infty
$$

$$
\sup _{0 \leq t<T} \sum_{n=-\infty}^{+\infty}\left\{\sum_{\ell=-\infty}^{n}\left[\dot{w}_{1}(\ell, t)+\dot{w}_{1}(\ell, t)\right]\right\}^{2}<+\infty
$$

$$
\sup _{0 \leq t<T} \sum_{n=-\infty}^{+\infty}\left[w_{1}^{2}(n, t)+w_{2}^{2}(n, t)\right]<+\infty .
$$

Let us prove item (c) (i.e. the boundedness of the potential energy). We can easily verify that whenever $\alpha(t) \in H(T)$, then

$$
\sum_{n=-\infty}^{+\infty}\left|\alpha_{j}(n, t)\right|^{p}\left|\alpha_{k}(n, t)\right|^{p} \leq C|| \alpha(\cdot) \|_{H}^{2 p}
$$

for any integer $p \geq 1$ and $1 \leq j, k \leq 2$. Let us rewrite

$$
I(t)=\sum_{n=-\infty}^{+\infty}\left[w_{1}^{2}(n, t)+w_{2}^{2}(n, t)\right]
$$

as

$$
I(t)=\gamma^{2} \sum_{j=1}^{6} A_{j}(t)
$$


where

$$
\begin{aligned}
A_{1}(t) & =\sum_{n=-\infty}^{+\infty}\left\{\sum_{\substack{\ell=-\infty \\
\ell \neq n, n+1}}^{+\infty} \int_{0}^{t} G_{1}(n-\ell, t-s) Q_{1}\left(\alpha_{1}, \alpha_{2}\right)(\ell, s) d s\right\}^{2}, \\
A_{2}(t) & =2 \sum_{n=-\infty}^{+\infty}\left\{\sum_{\substack{\ell=-\infty \\
\ell \neq n, n+1}}^{+\infty} \int_{0}^{t} G_{1}(n-\ell, t-s) Q_{1}\left(\alpha_{1}, \alpha_{2}\right)(\ell, s) d s\right\} \\
& \times\left\{\int_{0}^{t}\left[G_{1}(0, t-s) Q_{1}\left(\alpha_{1}, \alpha_{2}\right)(n, s)+G_{1}(-1, t-s) Q_{1}\left(\alpha_{1}, \alpha_{2}\right)(n+1, s)\right] d s\right\} \\
A_{3}(t)= & \sum_{n=-\infty}^{+\infty}\left\{\int_{0}^{t}\left[G_{1}(0, t-s) Q_{1}\left(\alpha_{1}, \alpha_{2}\right)(n, s)+G_{1}(-1, t-s) Q_{1}\left(\alpha_{1}, \alpha_{2}\right)(n+1, s)\right] d s\right\}^{2}, \\
A_{4}(t)= & \sum_{n=-\infty}^{+\infty}\left\{\sum_{\substack{\ell=-\infty \\
\ell \neq n, n+1}}^{+\infty} \int_{0}^{t} G_{2}(n-\ell, t-s) Q_{2}\left(\alpha_{1}, \alpha_{2}\right)(\ell, s) d s\right\} \\
A_{5}(t)= & 2 \sum_{n=-\infty}^{+\infty}\left\{\sum_{\substack{\ell=-\infty \\
\ell \neq n, n+1}}^{+\infty} \int_{0}^{t} G_{2}(n-\ell, t-s) Q_{2}\left(\alpha_{1}, \alpha_{2}\right)(\ell, s) d s\right\} \\
& \times\left\{\int_{0}^{t}\left[G_{2}(0, t-s) Q_{2}\left(\alpha_{1}, \alpha_{2}\right)(n, s)+G_{2}(1, t-s) Q_{2}\left(\alpha_{1}, \alpha_{2}\right)(n-1, s)\right] d s\right\} \\
A_{6}(t)= & \sum_{n=-\infty}^{+\infty}\left\{\int_{0}^{t}\left[G_{2}(0, t-s) Q_{2}\left(\alpha_{1}, \alpha_{2}\right)(n, s)+G_{2}(1, t-s) Q_{2}\left(\alpha_{1}, \alpha_{2}\right)(n-1, s)\right] d s\right\}^{2},
\end{aligned}
$$

$Q_{j}(j=1,2)$ are given by (3.9). Using (3.12) together with (2.14)-(2.16), we can obtain the estimates

$$
\begin{aligned}
& \sup _{0 \leq t<T}\left|A_{j}(t)\right| \leq C\left(T^{2}+T^{4}\right)^{2}\|\alpha(\cdot)\|_{H}^{2 p}, \quad j=1,2, \\
& \sup _{0 \leq t<T}\left|A_{j}(t)\right| \leq C\left(T^{2}+T^{4}\right) T^{2}\|\alpha(\cdot)\|_{H}^{2 p}, \quad j=3,4, \\
& \sup _{0 \leq t<T}\left|A_{j}(t)\right| \leq C T^{4}\|\alpha(\cdot)\|_{H}^{2 p}, \quad j=5,6,
\end{aligned}
$$

for some positive constant $C$. Clearly, (3.13) implies that

$$
\sup _{0 \leq t<T} I(t) \leq C\left(T^{2}+T^{4}\right)\|\alpha(\cdot)\|_{H}^{2 p},
$$

which proves item (c).

Next, let us consider item (b). For any $r \in \mathbb{Z}$, we have that

$$
\dot{w}_{1}(r, t)+\dot{w}_{2}(r, t)=\gamma \sum_{\ell=-\infty}^{+\infty} \int_{0}^{t} L(r, \ell, t, s) d s
$$


where

$$
\begin{aligned}
L(r, \ell, t, s) & =\alpha_{2}^{p}(\ell, s)\left\{m_{1} \dot{G}_{1}(r-\ell, t-s)+m_{2} \dot{G}_{1}(r-\ell+1, t-s)-\dot{G}_{2}(r-\ell, t-s)\right\} \\
& +\alpha_{1}^{p}(\ell, s)\left\{m_{1} \dot{G}_{2}(r-\ell, t-s)+m_{2} \dot{G}_{2}(r-\ell-1, t-s)-\dot{G}_{1}(r-\ell, t-s)\right\} .
\end{aligned}
$$

Consequently

$$
\begin{aligned}
& \sum_{r=-\infty}^{n}\left[\dot{w}_{1}(r, t)+\dot{w}_{2}(r, t)\right]=\gamma \sum_{r=-\infty}^{n} \sum_{\ell=-\infty}^{+\infty} \int_{0}^{t} L(r, \ell, t, s) d s \\
& =\gamma \sum_{r=-\infty}^{n} \sum_{\ell=-\infty}^{+\infty} \int_{0}^{t}\left[\dot{G}_{1}(r-\ell, t-s)\left\{m_{2} \alpha_{2}^{p}(\ell+1, s)+m_{1} \alpha_{2}^{p}(\ell, s)-\alpha_{1}^{p}(\ell, s)\right\}\right. \\
& \left.+\dot{G}_{2}(r-\ell, t-s)\left\{m_{2} \alpha_{1}^{p}(\ell-1, s)+m_{1} \alpha_{1}^{p}(\ell, s)-\alpha_{2}^{p}(\ell, s)\right\}\right] d s .
\end{aligned}
$$

In the above identity we can use the fact that $m_{1}+m_{2}=1$ together with the equalities

$$
\begin{aligned}
& \sum_{r=-\infty}^{n}\left[\dot{G}_{1}(r-\ell+1, t-s)-\dot{G}_{1}(r-\ell, t-s)\right]=\dot{G}_{1}(n-\ell+1, t-s), \\
& \sum_{r=-\infty}^{n}\left[\dot{G}_{2}(r-\ell-1, t-s)-\dot{G}_{2}(r-\ell, t-s)\right]=-\dot{G}_{2}(n-\ell, t-s)
\end{aligned}
$$

to obtain from (3.15) that

$$
\begin{aligned}
& \sum_{r=-\infty}^{n}\left[\dot{w}_{1}(r, t)+\dot{w}_{2}(r, t)\right] \\
& =\gamma m_{2} \sum_{\ell=-\infty}^{+\infty} \int_{0}^{t}\left[\dot{G}_{1}(n-\ell+1, t-s) \alpha_{2}^{p}(\ell, s)-\dot{G}_{2}(n-\ell, t-s) \alpha_{1}^{p}(\ell, s)\right] d s \\
& +\gamma \sum_{\ell=-\infty}^{+\infty} \sum_{r=-\infty}^{n} \int_{0}^{t}\left[\alpha_{2}^{p}(\ell, s)-\alpha_{1}^{p}(\ell, s)\right]\left[\dot{G}_{1}(r-\ell, t-s)-\dot{G}_{2}(r-\ell, t-s)\right] d s .
\end{aligned}
$$

We want to estimate the quantity

$$
\sup _{0 \leq t<T} \sum_{n=-\infty}^{+\infty}\left(\sum_{r=-\infty}^{n}\left[\dot{w}_{1}(r, t)+\dot{w}_{2}(r, t)\right]\right)^{2} .
$$

Let

$$
\begin{aligned}
I_{1}(n, t)= & \left(\sum_{\substack{\ell=-\infty \\
\ell \neq n+1, n+2}}^{+\infty} \int_{0}^{t} \dot{G}_{1}(n-\ell+1, t-s) \alpha_{2}^{p}(\ell, s) d s\right. \\
& \left.-\sum_{\substack{\ell=-\infty \\
\ell \neq n-1, n}}^{+\infty} \int_{0}^{t} \dot{G}_{2}(n-\ell, t-s) \alpha_{1}^{p}(\ell, s) d s\right)^{2}
\end{aligned}
$$


and

$$
\begin{aligned}
I_{2}(n, t) & =\left(\int _ { 0 } ^ { t } \left[\dot{G}_{1}(0, t-s) \alpha_{2}^{p}(n, s)+\dot{G}_{1}(1, t-s) \alpha_{2}^{p}(n-1, s)\right.\right. \\
& \left.\left.-\dot{G}_{2}(0, t-s) \alpha_{1}^{p}(n, s)-\dot{G}_{2}(-1, t-s) \alpha_{1}^{p}(n+1, s)\right] d s\right)^{2} .
\end{aligned}
$$

Using properties (2.20)-(2.22), it follows that

$I_{2}(n, t) \leq C\left(\int_{0}^{t}\left\{\left|\alpha_{2}(n, s)\right|^{p}+\left|\alpha_{1}(n, s)\right|^{p}+\left|\alpha_{2}(n-1, s)\right|^{p}+\left|\alpha_{1}(n+1, s)\right|^{p}\right\}|t-s|^{2} d s\right)^{2}$.

Since $\alpha \in H(T)$, it follows that

$$
\sup _{0 \leq t<T} \sum_{n=-\infty}^{+\infty} I_{2}(n, t) \leq C\|\alpha(\cdot)\|_{H}^{2 p} T^{6} .
$$

In order to get a bound for $I_{1}(n, t)$, we use (2.19) and observe that it follows from (2.11) that each $\dot{H}_{j}$ is a linear combination of $\cos (2 \sigma n) A(\sigma, t) / \Delta(\sigma), \cos (2 \sigma(n \pm 1)) A(\sigma, t) / \Delta(\sigma)$, and $\cos (2 \sigma n) A(\sigma, t)$, where $A(\sigma, t)=\cos \left(\omega_{-}(\sigma) t\right) \mp \cos \left(\omega_{+}(\sigma) t\right)$. We need to obtain a bound for

$$
\sum_{n=-\infty}^{+\infty}\left(\sum_{\substack{\ell=-\infty \\ \ell \neq n, n-1}}^{+\infty} \int_{0}^{t} \alpha_{1}^{p}(\ell, s) \dot{G}_{2}(n-\ell, t-s) d s\right)^{2}
$$

and

$$
\sum_{n=-\infty}^{+\infty}\left(\sum_{\substack{\ell=-\infty \\ \ell \neq n+1, n+2}}^{+\infty} \int_{0}^{t} \alpha_{2}^{p}(\ell, s) \dot{G}_{1}(n-\ell, t-s) d s\right)^{2}
$$

for any $0 \leq t<T$.

Let us consider the term

$$
P_{1}(n, t)=\int_{0}^{\pi / 2} F(\sigma, t) \cos (2 \sigma n) d \sigma
$$

where $F(\sigma, t)=\cos \left(\omega_{-}(\sigma) t\right) / \Delta(\sigma)$. Integration by parts twice gives us that

$$
P_{1}(n, t)=-\frac{1}{(n-\ell)^{2}} \int_{0}^{\pi / 2} \frac{\partial^{2} F}{\partial \sigma^{2}}(\sigma, t) \cos (2 \sigma n) d \sigma .
$$

Therefore

$$
\begin{aligned}
D_{1}(n, t) & =\left(\sum_{\substack{\ell=-\infty \\
\ell \neq n, n-1}}^{+\infty} \int_{0}^{t} \alpha_{1}^{p}(\ell, s) P_{1}(n-\ell, t-s) d s\right)^{2} \\
& =\left(\int_{0}^{t} \sum_{\substack{\ell=-\infty \\
\ell \neq n, n-1}}^{+\infty}\left\{\frac{\alpha_{1}^{p}(\ell, s)}{4(n-\ell)^{2}}\right\}\left\{\int_{0}^{\pi / 2} \frac{\partial^{2} F}{\partial \sigma^{2}}(\sigma, t-s) \cos (2 \sigma(n-\ell)) d \sigma\right\} d s\right)^{2} .
\end{aligned}
$$


Using Cauchy-Schwarz and Holder inequalities, we obtain that

$$
\begin{aligned}
D_{1}(n, t) \leq \int_{0}^{t} & \sum_{\substack{\ell=-\infty \\
\ell \neq n, n-1}}^{+\infty} \frac{\alpha_{1}^{2 p}(\ell, s)}{16(n-\ell)^{4}} d s \\
& \times \int_{0}^{t} \sum_{\substack{\ell=-\infty \\
\ell \neq n, n-1}}^{+\infty}\left(\int_{0}^{\pi / 2} \frac{\partial^{2} F}{\partial \sigma^{2}}(\sigma, t-s) \cos (2 \sigma(n-\ell)) d \sigma\right)^{2} d s .
\end{aligned}
$$

Consequently

$$
\begin{aligned}
& \sum_{n=-\infty}^{+\infty} D_{1}(n, t) \leq\left(\int_{0}^{t} \sum_{\substack{\ell=-\infty \\
\ell \neq 0}}^{+\infty} \frac{1}{16 \ell^{2}}\left(\sum_{m=-\infty}^{+\infty} \alpha_{1}^{2 p}(m, s)\right) d s\right) \\
& \times\left(\int_{0}^{t} \sum_{\ell=-\infty}^{+\infty}\left[\int_{0}^{\pi / 2} \frac{\partial^{2} F}{\partial \sigma^{2}}(\sigma, t-s) \cos (2 \sigma(n-\ell)) d \sigma\right]^{2} d s\right) \\
& \leq\|\alpha(\cdot)\|_{H}^{2 p} \frac{\pi^{4}|t|}{720} \int_{0}^{t} \sum_{\ell=-\infty}^{+\infty} K_{\ell}^{2}(t-s) d s
\end{aligned}
$$

where

$$
K_{n}(t)=\int_{0}^{\pi / 2} \frac{\partial^{2} F}{\partial \sigma^{2}}(\sigma, t) \cos (2 \sigma n) d \sigma
$$

are the Fourier coefficients of the function $\frac{\partial^{2} F}{\partial \sigma^{2}}$ and we used that $\sum_{m=1}^{+\infty} m^{-4}=\pi^{4} / 90$. Clearly $\frac{\partial^{2} F}{\partial \sigma^{2}}(\cdot, t) \in L^{2}(0, \pi / 2)$ and it is an even function in $\sigma$. Therefore Parseval's inequality applies and

$$
\sum_{\ell=-\infty}^{+\infty} A_{t}^{2}(t) \leq\left\|\frac{\partial^{2} F}{\partial \sigma^{2}}(\cdot, t)\right\|_{L^{2}}^{2} .
$$

Thus, (3.21) gives us an estimate for

$$
\sup _{0 \leq t<T} \sum_{m=-\infty}^{+\infty} D_{1}(n, t) \leq C\|\alpha(\cdot)\|_{H}^{2 p}\left(T^{2}+T^{4}+T^{6}\right)
$$

for some positive constant $C$.

All other terms included in $\dot{H}_{j}(n, t, \sigma)$ can be treated similarly to the above discussion to conclude that

$$
\sup _{0 \leq t<T} \sum_{m=-\infty}^{+\infty} I_{1}(n, t) \leq \widetilde{C}\|\alpha(\cdot)\|_{H}^{2 p}\left(T^{2}+T^{4}+T^{6}\right)
$$

for some positive constant $\widetilde{C}$. 
It remains to get a bound for the term $\sup _{0 \leq t<T} \sum_{n=-\infty}^{+\infty} I_{3}^{2}(n, t)$ where

$$
I_{3}(n, t)=\sum_{\ell=-\infty}^{+\infty} \sum_{r=-\infty}^{n} \int_{0}^{t}\left[\alpha_{2}^{p}(\ell, s)-\alpha_{1}^{p}(\ell, s)\right]\left[\dot{G}_{1}(r-\ell, t-s)-\dot{G}_{2}(r-\ell, t-s)\right] d s .
$$

Let us write $I_{3}(n, t)=J_{1}(n, t)+J_{n}(n, t)$ where

$$
J_{1}(n, t)=\sum_{\ell=-\infty}^{+\infty} \sum_{\substack{r=-\infty \\ r \neq \ell-1, \ell+1}}^{n} \int_{0}^{t}\left[\alpha_{1}^{p}(\ell, s)-\alpha_{1}^{p}(\ell, s)\right]\left[\dot{G}_{1}(r-\ell, t-s)-\dot{G}_{2}(r-\ell, t-s)\right] d s .
$$

This term can be treated as follows: Due to Condition 1 we have

$$
\begin{aligned}
& \dot{G}_{12}(r, t) \equiv \dot{G}_{1}(r, t)-\dot{G}_{2}(r, t)=\frac{1}{\pi} \int_{0}^{\pi / 2}\left[\dot{H}_{1}(r, t, \sigma)-\dot{H}_{2}(r, t, \sigma)\right] d \sigma \\
= & \frac{m_{2}}{2 \pi} \int_{0}^{\pi / 2} \frac{1}{\Delta(\sigma)}\left[\cos \left(\omega_{-}(\sigma) t\right)-\cos \left(\omega_{+}(\sigma) t\right)\right][\cos (2 \sigma(r+1))-\cos (2 \sigma(r-1))] d \sigma .
\end{aligned}
$$

Consequently, $\dot{G}_{12}(r, t)$ as a function of $r$ is an odd function. This implies that

$$
\sum_{\substack{r=-\infty \\ r \neq \pm 1}}^{n} \dot{G}_{12}(r, t)=\sum_{\substack{r=-\infty \\ r \neq-1}}^{M(n)} \dot{G}_{12}(r, t)
$$

where

$$
M(n)=\left\{\begin{array}{lll}
-n-1 & \text { if } & n>1 \\
-2 & \text { if } & n=0, \pm 1, \\
n & \text { if } & n<-1 .
\end{array}\right.
$$

Let $F_{1}(\sigma, t)$ be defined as

$$
F_{1}(\sigma, t)=\frac{\cos \left(\omega_{-}(\sigma) t\right)-\cos \left(\omega_{+}(\sigma) t\right)}{\Delta(\sigma)} .
$$

Therefore

$$
\dot{G}_{12}(n, t)=\frac{m_{2}}{2 \pi} \int_{0}^{2 \pi}[\cos (2 \sigma(n+1))-\cos (2 \sigma(n-1))] F_{1}(\sigma, t) d \sigma .
$$

Integration by parts (three times) give us that

$$
\begin{aligned}
\sum_{\substack{r=-\infty \\
r \neq \ell+1, \ell-1}}^{M(n-\ell)} \dot{G}_{12}(r-\ell, t) & =\frac{m_{2}}{2 \pi} \sum_{\substack{r=-\infty \\
r \neq \ell-1}}^{M(n-\ell)} \frac{1}{8(r-\ell+1)^{3}} \int_{0}^{2 \pi} \sin (2 \sigma(r-\ell+1)) \frac{\partial^{3} F_{1}(\sigma, t)}{\partial \sigma^{3}} d \sigma \\
& -\frac{m_{2}}{2 \pi} \sum_{\substack{r=-\infty \\
r \neq \ell+1}}^{M(n-\ell)} \frac{1}{8(r-\ell-1)^{3}} \int_{0}^{2 \pi} \sin (2 \sigma(r-\ell-1)) \frac{\partial^{3} F_{1}(\sigma, t)}{\partial \sigma^{3}} d \sigma .
\end{aligned}
$$


Consequently, we obtain the estimate

$$
\begin{aligned}
& \left|\sum_{\ell=-\infty}^{+\infty} \int_{0}^{t}\left[\alpha_{2}^{p}(\ell, s)-\alpha_{1}^{p}(\ell, s)\right] \sum_{\substack{r=-\infty \\
r \neq 1, \ell-1}}^{M(n-\ell)} \dot{G}_{12}(r-\ell, t-s) d s\right| \\
& \leq C \sum_{\ell=-\infty}^{+\infty} \int_{0}^{t}\left|\alpha_{2}^{p}(\ell, s)-\alpha_{1}^{p}(\ell, s)\right| d s \sum_{r=-\infty}^{M(n)} \frac{1}{|r|^{3}} \\
& \leq\left. C|| \alpha(\cdot)\right|_{H} ^{p}\left(T+T^{2}+T^{3}\right) \sum_{r=-\infty}^{M(n)} \frac{1}{|r|^{3}}
\end{aligned}
$$

for any $0 \leq t<T$ where $C$ is a positive constant, where we used

$$
\left|\int_{0}^{2 \pi} \sin (2 \sigma(r-\ell \pm 1)) \frac{\partial^{3} F_{1}(\sigma, t)}{\partial \sigma^{3}} d \sigma\right| \leq C\left[1+|t-s|+|t-s|^{2}\right] .
$$

Thus (3.24) implies that

$$
\left|J_{1}(n, t)\right|^{2} \leq C \|\left.\alpha(\cdot)\right|_{H} ^{2 p}\left(\sum_{r=-\infty}^{M(n)} \frac{1}{|r|^{3}}\right)^{2}\left(T+T^{2}+T^{3}\right)^{2} .
$$

Using the comparison criteria with integrals, we have that

$$
\sum_{r=-\infty}^{M(n)} \frac{1}{|r|^{3}} \leq \frac{1}{2 M^{2}(n)}, \quad n \neq 0, \pm 1 .
$$

Consequently, we obtain that

$$
\sum_{m=-\infty}^{+\infty} J_{1}^{2}(n, t) \leq C\|\alpha(\cdot)\|_{H}^{2 p}\left(T+T^{2}+T^{3}\right)^{2} .
$$

Therefore

$$
\sup _{0 \leq t<T} \sum_{n=-\infty}^{+\infty} J_{1}^{2}(n, t)<+\infty
$$

Since $\dot{G}_{12}(n, t)$ is an odd function of $n, J_{2}(n, t) \equiv 0$. Consequently, from (3.25) it follows that

$$
\sup _{0 \leq t<T} \sum_{n=-\infty}^{+\infty} I_{3}^{2}(n, t) \leq C\|\alpha(\cdot)\|_{H}^{2 p}\left(T^{2}+T^{4}+T^{6}\right) .
$$

From (3.20), (3.23) and (3.26) we conclude that

$$
\sup _{0 \leq t<T} \sum_{n=-\infty}^{+\infty}\left(\sum_{r=-\infty}^{n}\left[\dot{w}_{1}(r, t)+\dot{w}_{2}(r, t)\right]\right)^{2} \leq C\|\alpha(\cdot)\|_{H}^{2 p}\left(T^{2}+T^{4}+T^{6}\right) .
$$

The proof of item (a) (see a few lines above (3.12)) follows the same sequence of ideas described in item (b); therefore we will omit the proof. It follows from this estimate that taking $T>0$ sufficiently small, the map $P$ defined by (3.12) sends the closed subset $F_{R}$ into itself. 
Now we want to prove that $P$ is a contraction map from $F_{R}$ if $T>0$ is chosen small enough. Let $\alpha(t), \delta(t) \in F_{R}(T)$. Thus

$$
\begin{aligned}
& P \alpha(t)=\left(\cdots ; \widetilde{P}_{1} \alpha_{1}(j-1, t), \widetilde{P}_{2} \alpha_{2}(j-1, t) ; \widetilde{P}_{1} \alpha_{1}(j, t), \widetilde{P}_{2} \alpha_{2}(j, t) ; \cdots\right), \\
& P \delta(t)=\left(\cdots ; \widetilde{P}_{1} \delta_{1}(j-1, t), \widetilde{P}_{2} \delta_{2}(j-1, t) ; \widetilde{P}_{1} \delta_{1}(j, t), \widetilde{P}_{2} \delta_{2}(j, t) ; \cdots\right)
\end{aligned}
$$

where

$$
\begin{aligned}
& \widetilde{P}_{k} \alpha_{k}(n, t)=\beta_{k}(n, t)+\gamma \sum_{\ell=-\infty}^{+\infty} \int_{0}^{t} G_{k}(n-\ell, t-s) Q_{k}\left(\alpha_{1}, \alpha_{2}\right)(\ell, s) d s, \\
& \widetilde{P}_{k} \delta_{k}(n, t)=\beta_{k}(n, t)+\gamma \sum_{\ell=-\infty}^{+\infty} \int_{0}^{t} G_{k}(n-\ell, t-s) Q_{k}\left(\delta_{1}, \delta_{2}\right)(\ell, s) d s
\end{aligned}
$$

with $k=1,2$ and $Q_{k}$ as in (3.9).

Let us define $\tilde{w}_{1}(n, t)$ and $\tilde{w}_{2}(n, t)$ as

$$
\begin{aligned}
\tilde{w}_{1}(n, t) & =\widetilde{P}_{1} \alpha_{1}-\widetilde{P}_{1} \delta_{1} \\
& =\gamma \sum_{\ell=-\infty}^{+\infty} \int_{0}^{t} G_{1}(n-\ell, t-s)\left[Q_{1}\left(\alpha_{2}, \alpha_{2}\right)(\ell, s)-Q_{1}\left(\delta_{1}, \delta_{2}\right)(\ell, s)\right] d s, j=1,2 .
\end{aligned}
$$

In order to estimate $\|P \alpha-P \delta\|_{H}^{2}$, we begin by the term

$$
I(t)=\sum_{n=-\infty}^{+\infty}\left[\tilde{w}_{1}^{2}(n, t)+\tilde{w}_{2}^{2}(n, t)\right]
$$

and we write it as

$$
I(t)=\gamma^{2} \sum_{j=1}^{6} B_{j}(t)
$$


where

$$
\begin{aligned}
B_{1}(t)= & \sum_{n=-\infty}^{+\infty}\left\{\sum_{\substack{\ell=-\infty \\
\ell \neq n, n+1}}^{+\infty} \int_{0}^{t} G_{1}(n-\ell, t-s)\left[Q_{1}\left(\alpha_{1}, \alpha_{2}\right)-Q_{1}\left(\delta_{1}, \delta_{2}\right)\right](\ell, s) d s\right\}^{2}, \\
B_{2}(t)= & 2 \sum_{n=-\infty}^{+\infty}\left\{\sum_{\substack{\ell=-\infty \\
\ell \neq n, n+1}}^{+\infty} \int_{0}^{t} G_{1}(n-\ell, t-s)\left[Q_{1}\left(\alpha_{1}, \alpha_{2}\right)-Q_{1}\left(\delta_{1}, \delta_{2}\right)\right](\ell, s) d s\right\} \\
& \times\left\{\int _ { 0 } ^ { t } \left[G_{1}(0, t-s)\left[Q_{1}\left(\alpha_{1}, \alpha_{2}\right)-Q_{1}\left(\delta_{1}, \delta_{2}\right)\right](n, s)\right.\right. \\
& \left.\left.+G_{1}(-1, t-s)\left[Q_{1}\left(\alpha_{1}, \alpha_{2}\right)-Q_{1}\left(\delta_{1}, \delta_{2}\right)\right](n+1, s)\right] d s\right\} \\
B_{3}(t)= & \left\{\int _ { 0 } ^ { t } \left[G_{1}(0, t-s)\left[Q_{1}\left(\alpha_{1}, \alpha_{2}\right)-Q_{1}\left(\delta_{1}, \delta_{2}\right)\right](n, s)\right.\right. \\
& \left.\left.+G_{1}(-1, t-s)\left[Q_{1}\left(\alpha_{1}, \alpha_{2}\right)-Q_{1}\left(\delta_{1}, \delta_{2}\right)\right](n+1, s)\right] d s\right\}^{2}, \\
B_{4}(t)= & \sum_{n=-\infty}^{+\infty}\left\{\sum_{\substack{\ell=-\infty \\
\ell \neq n, n-1}}^{+\infty} \int_{0}^{t} G_{2}(n-\ell, t-s)\left[Q_{1}\left(\alpha_{1}, \alpha_{2}\right)-Q_{2}\left(\delta_{1}, \delta_{2}\right)\right](\ell, s) d s\right\} \\
& \left.\left.+G_{2}(1, t-s)\left[Q_{2}\left(\alpha_{1}, \alpha_{2}\right)-Q_{2}\left(\delta_{1}, \delta_{2}\right)\right](n-1, s)\right] d s\right\}^{2} \\
B_{6}(t)= & \left\{\int_{0}^{t}\left[G_{2}(0, t-s)\left[Q_{2}\left(\alpha_{1}, \alpha_{2}\right)-Q_{2}\left(\delta_{1}, \delta_{2}\right)\right](n, s)\right]\right. \\
B_{5}(t)= & 2 \sum_{n=-\infty}^{+\infty}\left\{\sum_{\substack{\ell=-\infty \\
\ell \neq n, n-1}}^{+\infty} \int_{0}^{t} G_{2}(n-\ell, t-s)\left[Q_{1}\left(\alpha_{1}, \alpha_{2}\right)-Q_{1}\left(\delta_{1}, \delta_{2}\right)\right](\ell, s) d s\right\} \\
& \int_{0}(0, t-s)\left[Q_{2}\left(\alpha_{1}, \alpha_{2}\right)-Q_{2}\left(\delta_{1}, \delta_{2}\right)\right](n, s)
\end{aligned}
$$

We can also write

$$
\begin{gathered}
{\left[Q_{1}\left(\alpha_{1}, \alpha_{2}\right)-Q_{1}\left(\delta_{1}, \delta_{2}\right)\right](\ell, s)=m_{2}\left[\alpha_{2}(\ell+1, s)-\delta_{1}(\ell+1, s)\right] g_{2}(\ell+1, s)} \\
+m_{1}\left[\alpha_{2}(\ell, s)-\delta_{2}(\ell, s)\right] g_{2}(\ell, s)-\left[\alpha_{1}(\ell, s)-\delta_{1}(\ell, s)\right] g_{1}(\ell, s) \\
{\left[Q_{2}\left(\alpha_{1}, \alpha_{2}\right)-Q_{2}\left(\delta_{1}, \delta_{2}\right)\right](\ell, s)=m_{2}\left[\alpha_{1}(\ell-1, s)-\delta_{1}(\ell-1, s)\right] g_{1}(\ell-1, s)} \\
+m_{1}\left[\alpha_{1}(\ell, s)-\delta_{1}(\ell, s)\right] g_{1}(\ell, s)-\left[\alpha_{2}(\ell, s)-\delta_{2}(\ell, s)\right] g_{2}(\ell, s)
\end{gathered}
$$


where $(j=1,2)$

$$
\begin{aligned}
g_{j}(\ell, s) & =\left[\alpha_{j}(\ell, s)\right]^{p-1}+\left[\alpha_{j}(\ell, s)\right]^{p-2}\left[\delta_{j}(\ell, s)\right] \\
& +\cdots+\left[\alpha_{j}(\ell, s)\right][\delta(\ell, s)]^{p-2}+\left[\delta_{j}(\ell, s)\right]^{p-1} .
\end{aligned}
$$

We observe that since $\alpha, \delta \in H(T)$, the inequality

$$
\sum_{n=-\infty}^{+\infty}\left|\alpha_{j}(n, t)\right|^{q}\left|\delta_{j}(n, t)\right|^{q} \leq C\|\alpha\|_{H}^{q}\|\delta\|_{H}^{q}
$$

holds for any integer $q \geq 1, j, k=1,2$ and $0 \leq t<T$. Also, since $\alpha$ and $\delta$ belong to $F_{R}$, then in particular $\|\alpha\|_{H} \leq R+\|\beta\|_{H}$ and $\|\delta\|_{H} \leq R+\|\beta\|_{H}$ where the components of $\beta$ solve problem (2.9) (with $\kappa=1$ in our case). Clearly $\|\beta\|_{H} \leq \widetilde{C}$ where $\widetilde{C}$ is a positive constant depending only on the initial data at $t=0$.

Using (3.30)-(3.33) together with (2.14)-(2.16), we obtain the estimates

$$
\begin{aligned}
\sup _{0 \leq t<T}\left|B_{j}(t)\right| & \leq C\left(T^{2}+T^{4}\right)^{2}\|\alpha-\delta\|_{H}^{2}\left(\|\alpha\|_{H}+\|\delta\|_{H}\right)^{2(p-1)} \\
& \leq C\left(T^{2}+T^{4}\right)^{2}\|\alpha-\delta\|_{H}^{2}\left(2\|\beta\|_{H}+2 R\right)^{2(p-1)}
\end{aligned}
$$

for $j=1,2,5,6$ and

$$
\sup _{0 \leq t<T}\left|B_{j}(t)\right| \leq C\left(T^{2}+T^{4}\right) T^{2}\|\alpha-\delta\|_{H}^{2}\left(2|| \beta \|_{H}+2 R\right)^{2(p-1)}
$$

for $j=3,4$. Consequently

$$
\begin{aligned}
\sup _{0 \leq t<T} I(t) & =\sup _{0 \leq t<T} \sum_{n=-\infty}^{+\infty}\left[\tilde{w}_{1}^{2}(n, t)+\tilde{w}_{2}^{2}(n, t)\right] \\
& \leq C\left(T^{2}+T^{4}\right) T^{2}\|\alpha-\delta\|_{H}^{2}\left(\|\beta\|_{H}+R\right)^{2(p-1)}
\end{aligned}
$$

for some positive constant $C$.

The remaining estimates for $\|P \alpha-P \delta\|_{H}$ will follow almost the same sequence of ideas we used while showing that $P$ maps $F_{R}$ into itself. We just indicate the steps and omit details.

From (3.28) we obtain that

$$
\begin{aligned}
& \sum_{r=-\infty}^{n}\left[\dot{\tilde{w}}_{1}^{2}(r, t)+\dot{\tilde{w}}_{2}^{2}(r, t)\right] \\
& \quad=\gamma \sum_{r=-\infty}^{n} \sum_{\ell=-\infty}^{+\infty} \int_{0}^{t}\left[\dot{G}_{1}(r-\ell, t-s) Q_{11}(\ell, s)+\dot{G}_{2}(r-\ell, t-s) Q_{22}(\ell, s)\right] d s
\end{aligned}
$$

where

$$
\begin{aligned}
Q_{11}(\ell, s) & =m_{2}\left[\alpha_{2}(\ell+1, s)-\delta_{2}(\ell+1, s)\right] g_{2}(\ell+1, s) \\
& +m_{1}\left[\alpha_{2}(\ell, s)-\delta_{2}(\ell, s)\right] g_{2}(\ell, s)-\left[\alpha_{1}(\ell, s)-\delta_{1}(\ell, s)\right] g_{1}(\ell, s), \\
Q_{22}(\ell, s) & =m_{2}\left[\alpha_{1}(\ell-1, s)-\delta_{1}(\ell-1, s)\right] g_{1}(\ell-1, s) \\
& +m_{1}\left[\alpha_{1}(\ell, s)-\delta_{1}(\ell, s)\right] g_{1}(\ell, s)-\left[\alpha_{2}(\ell, s)-\delta_{2}(\ell, s)\right] g_{2}(\ell, s)
\end{aligned}
$$


where $g_{j}(j=1,2)$ are as in $(3.32)$. Now we use the relation $m_{1}+m_{2}=1$ and the identities (3.16), (3.17) to obtain

$$
\begin{gathered}
\sum_{r=-\infty}^{n}\left[\dot{\tilde{w}}_{1}^{2}(r, t)+\dot{\tilde{w}}_{2}^{2}(r, t)\right] \\
=\gamma m_{2} \sum_{\ell=-\infty}^{+\infty} \int_{0}^{t}\left[\dot{G}_{1}(n-\ell+1, t-s)\left(\alpha_{2}(\ell, s)-\delta_{2}(\ell, s)\right) g_{2}(\ell, s)\right. \\
\left.-\dot{G}_{2}(n-\ell, t-s)\left(\alpha(\ell, s)-\delta_{1}(\ell, s)\right) g_{1}(\ell, s)\right] d s \\
+\gamma \sum_{r=-\infty}^{n} \sum_{\ell=-\infty}^{+\infty} \int_{0}^{t}\left[\left(\alpha_{2}(\ell, s)-\delta_{2}(\ell, s)\right) g_{2}(\ell, s)-\left(\alpha_{1}(\ell, s)-\delta_{1}(\ell, s)\right) g_{1}(\ell, s)\right] \\
\times\left[\dot{G}_{1}(r-\ell, t-s)-\dot{G}_{2}(r-\ell, t-s)\right] d s .
\end{gathered}
$$

Now we can proceed in the same manner we did in order to obtain (3.27). We get

$$
\begin{gathered}
\sup _{0 \leq t<T} \sum_{n=-\infty}^{+\infty}\left(\sum_{r=-\infty}^{n}\left[\dot{\tilde{w}}_{1}^{2}(r, t)+\dot{\tilde{w}}_{2}^{2}(r, t)\right]\right)^{2} \\
\leq C\|\alpha-\delta\|_{H}^{2}\left(\|\alpha\|_{H}+\|\delta\|_{H}\right)^{2(p-1)}\left(T^{2}+T^{4}+T^{6}\right) \\
\leq\|\alpha-\delta\|_{H}^{2}\left(2\|\beta\|_{H}+2 R\right)^{2(p-1)}\left(T^{2}+T^{4}+T^{6}\right)
\end{gathered}
$$

for some positive constant $C$.

Similar analysis gives us the same bound as above for the term

$$
\sup _{0 \leq t<T} \sum_{n=-\infty}^{+\infty}\left(\sum_{r=-\infty}^{n}\left[\dot{\tilde{w}}_{1}^{2}(r, t)+\dot{\tilde{w}}_{2}^{2}(r-1, t)\right]\right)^{2} .
$$

Consequently, this information together with (3.34) and (3.35) shows that by choosing $T>0$ small enough so that

$$
3 C\left(2\|\beta\|_{H}+2 R\right)^{2(p-1)}\left(T^{2}+T^{4}+T^{6}\right)<1,
$$

$P$ will be a contraction in $F_{R}$. Hence we deduce that there exists a unique fixed point $\tilde{\alpha} \in F_{R}$, that is,

$$
P_{j} \tilde{\alpha}_{j}(n, t)=\tilde{\alpha}_{j}(n, t), \quad j=1,2,
$$

for all $n$ and all $0 \leq t<T$. This pair $\left\{\tilde{\alpha}_{1}, \tilde{\alpha}_{2}\right\}$ is the unique solution of (3.4), (3.5) (or equivalently of (2.7), (2.8) with $k=1)$. This concludes the proof of Theorem 3.1.

Zorn's lemma can be applied to our case. Thus the local solution $\tilde{\alpha}(t)$ we found above can be extended to the maximal interval of existence $0 \leq t<T_{\max }$. In the next section we want to prove that $T_{\max }=+\infty$.

4. Global Existence. In this section we want to prove that the local solution of problem (2.7), (2.8) we found in Theorem 3.1 can be continued for all time, that is, 
$T_{\max }=\infty$. To do this, we need an a priori estimate. Under the assumptions of Theorem 3.1 we consider

$$
\begin{aligned}
E(t)=\frac{1}{2} & \sum_{n=-\infty}^{+\infty}\left\{\frac{1}{m_{1}}\left(\sum_{\ell=-\infty}\left[\dot{\alpha}_{2}(\ell, t)+\dot{\alpha}_{1}(\ell-1, t)\right]\right)^{2}\right. \\
& \left.+\frac{1}{m_{2}}\left(\sum_{\ell=-\infty}^{n}\left[\dot{\alpha}_{2}(\ell, t)+\dot{\alpha}_{1}(\ell, t)\right]\right)^{2}\right\} \\
& +\frac{1}{2} \sum_{n=-\infty}^{+\infty}\left[\alpha_{1}^{2}(n, t)+\alpha_{2}^{2}(n, t)\right]+\frac{\gamma}{p+1} \sum_{n=-\infty}^{+\infty}\left[\alpha_{1}^{p+1}(n, t)+\alpha_{2}^{p+1}(n, t)\right] .
\end{aligned}
$$

Observe that the term $\sum_{n=-\infty}^{+\infty}\left[\alpha_{1}^{p+1}(n, t)+\alpha_{2}^{p+1}(n, t)\right]$ is finite for each $t$ in the interval of existence because

$$
\alpha(t)=\left(\cdots ; \alpha_{1}(j-1, t), \alpha_{2}(j-1, t) ; \alpha_{1}(j, t), \alpha_{2}(j, t) ; \alpha_{1}(j+1, t), \alpha_{2}(j+1, t) ; \cdots\right)
$$

belongs to $H(T)$ (for $T<T_{\max }$ ). In fact

$$
\sum_{n=-\infty}^{+\infty}\left[\alpha_{1}^{p+1}(n, t)+\alpha_{2}^{p+1}(n, t)\right] \leq 2\|\alpha(\cdot)\|_{H}^{p+1} .
$$

We shall refer to $E(t)$ as the total energy associated with problem (2.7), (2.8).

Theorem 4.1 (Global existence and uniqueness). Let $p \geq 3$ be an odd integer and $\gamma>0$. Suppose that the initial conditions for (2.7), (2.8) satisfy assumption (3.2), Conditions 1 and 2 , and

$$
\sum_{n=-\infty}^{+\infty}\left(\left|\tilde{a}_{1}(n)\right|^{p+1}+\left|\tilde{a}_{2}(n)\right|^{p+1}\right)<+\infty .
$$

Then, the finite energy solution $\left(\alpha_{1}(n, t), \alpha_{2}(n, t)\right)$ of problem $(2.7),(2.8)$ with initial conditions $\left(\tilde{a}_{1}(n), \tilde{a}_{2}(n)\right)$ exists globally and it is unique.

Proof. Let $\alpha(t)$ be as in (4.2) in an interval $0 \leq T<T_{\max }$ with $T$ "near" $T_{\max }$. Using equations (2.7) and (2.8), we can verify that

$$
\frac{d E(t)}{d t}=0 \text { for any } 0 \leq t \leq T,
$$

that is, $E(t)=E(0)$ in this interval. In particular this gives us the a priori estimate we needed, that is,

$$
\begin{gathered}
\sum_{n=-\infty}^{+\infty}\left\{\left(\sum_{\ell=-\infty}^{n}\left[\dot{\alpha}_{2}(\ell, t)+\dot{\alpha}_{1}(\ell-1, t)\right]\right)^{2}+\left(\sum_{\ell=-\infty}^{n}\left[\dot{\alpha}_{2}(\ell, t)+\dot{\alpha}_{1}(\ell, t)\right]\right)^{2}\right\} \\
+\sum_{n=-\infty}^{+\infty}\left[\alpha_{1}^{2}(n, t)+\alpha_{2}^{2}(n, t)\right] \leq C<+\infty
\end{gathered}
$$

for any $0 \leq t<T$ and some positive constant $C$ which depends only on the initial data. Thus we must have that $T_{\max }=+\infty$. Now we prove the uniqueness of the solution. 
Suppose that there exist two solutions $\left(\alpha_{1}(n, t), \alpha_{2}(n, t)\right)$ and $\left(\delta_{1}(n, t), \delta_{2}(n, t)\right)$ of $(3.4)$, (3.5) with the same initial conditions

$$
\alpha_{j}(n, 0)=\delta_{j}(n, 0), \quad \dot{\alpha}_{j}(n, 0)=\dot{\delta}_{j}(n, 0)
$$

Let us introduce

$$
\begin{aligned}
& u_{1}(n, t)=\sum_{\ell=-\infty}^{n}\left[\alpha_{2}(k, t)+\alpha_{1}(k-1, t)\right], \\
& u_{2}(n, t)=\sum_{\ell=-\infty}^{n}\left[\alpha_{2}(k, t)+\alpha_{1}(k, t)\right], \\
& w_{1}(n, t)=\sum_{\ell=-\infty}^{n}\left[\delta_{2}(k, t)+\delta_{1}(k-1, t)\right], \\
& w_{2}(n, t)=\sum_{\ell=-\infty}^{n}\left[\delta_{2}(k, t)+\delta_{1}(k, t)\right],
\end{aligned}
$$

as well as

$$
z_{j}(n, t)=u_{j}(n, t)-w_{j}(n, t), \quad j=1,2 .
$$

Observe that substitution of $\left(\alpha_{1}(n, t), \alpha_{2}(n, t)\right)$ by the expressions (3.4), (3.5) shows that $u_{j}(n, t)$ and $w_{j}(n, t)(j=1,2)$ are well defined and bounded.

Clearly $z_{j}(n, 0)=0$. We claim that

$$
z_{j}(n, t) \equiv 0, \quad \forall t \in[0, T]
$$

Indeed the system of equations for $z_{j}(n, t)$ can be written in the form

$$
\begin{aligned}
& \frac{1}{m_{1}} \ddot{z}_{1}(n, t)+z_{1}(n, t)-z_{2}(n, t)+z_{1}(n, t)-z_{2}(n-1, t) \\
&=-\gamma\left[z_{1}(n, t)-z_{2}(n, t)\right] h_{1}(n, t)-\gamma\left[z_{1}(n, t)-z_{2}(n-1, t)\right] h_{2}(n, t), \\
& \frac{1}{m_{2}} \ddot{z}_{2}(n, t)+z_{2}(n, t)-z_{1}(n, t)+z_{2}(n, t)-z(n+1, t) \\
&=-\gamma\left[z_{1}(n, t)-z_{2}(n, t)\right] h_{1}(n, t)-\gamma\left[z_{1}(n+1, t)-z_{2}(n, t)\right] h_{2}(n+1, t)
\end{aligned}
$$

where

$$
\begin{aligned}
& h_{1}(n, t)=[A(n, t)]^{p-1}+[A(n, t)]^{p-2} B(n, t)+\cdots+A(n, t)[B(n, t)]^{p-2}+[B(n, t)]^{p-1}, \\
& h_{2}(n, t)=[C(n, t)]^{p-1}+[C(n, t)]^{p-2} D(n, t)+\cdots+C(n, t)[D(n, t)]^{p-2}+[D(n, t)]^{p-1}
\end{aligned}
$$

with

$$
\begin{array}{ll}
A(n, t)=u_{1}(n, t)-u_{2}(n, t), & B(n, t)=w_{1}(n, t)-w_{2}(n, t), \\
C(n, t)=u_{1}(n, t)-u_{2}(n-1, t), & D(n, t)=w_{1}(n, t)-w_{2}(n-1, t) .
\end{array}
$$


Let $\varphi(t)$ be defined as

$$
\begin{aligned}
\varphi(t)=\sum_{n=-\infty}^{+\infty}\left\{\frac{1}{2 m_{1}}\left[\dot{z}_{1}(n, t)\right]^{2}\right. & +\frac{1}{2 m_{2}}\left[\dot{z}_{2}(n, t)\right]^{2}+\frac{1}{2}\left[z_{1}(n, t)-z_{2}(n, t)\right]^{2} \\
& \left.+\frac{1}{2}\left[z_{1}(n, t)-z_{2}(n-1, t)\right]^{2}\right\} \geq 0 .
\end{aligned}
$$

Due to the identities (4.3), (4.4) we deduce that $\varphi(t)$ satisfies

$$
\begin{aligned}
\frac{d \varphi(t)}{d t}= & -\gamma \sum_{n=-\infty}^{+\infty}\left\{\left[\dot{z}_{1}(n, t)+\dot{z}_{2}(n, t)\right]\left[z_{1}(n, t)-z_{2}(n, t)\right] h_{1}(n, t)\right. \\
& \left.+\left[\dot{z}_{1}(n, t)+\dot{z}_{2}(n-1, t)\right]\left[z_{1}(n, t)-z_{2}(n-1, t)\right] h_{2}(n, t)\right\}
\end{aligned}
$$

from which the inequality

$$
\begin{aligned}
\left|\frac{d \varphi(t)}{d t}\right| & \leq \frac{\gamma}{2}\left\{\sup _{-\infty<n<+\infty}\left|h_{1}(n, t)\right| \sum_{n=-\infty}^{+\infty}\left(\left[\dot{z}_{1}(n, t)\right]^{2}+\left[\dot{z}_{2}(n, t)\right]^{2}+2\left[z_{1}(n, t)-z_{2}(n, t)\right]^{2}\right)\right. \\
& \left.+\sup _{-\infty<n<+\infty}\left|h_{2}(n, t)\right| \sum_{n=-\infty}^{+\infty}\left(\left[\dot{z}_{1}(n, t)\right]^{2}+\left[\dot{z}_{2}(n, t)\right]^{2}+2\left[z_{1}(n, t)-z_{2}(n-1, t)\right]^{2}\right)\right\} \\
& \leq C \varphi(t)
\end{aligned}
$$

follows. Using Gronwall's lemma, we deduce that $\varphi(t)$ is identically zero. Therefore $u_{j}(n, t)=w_{j}(n, t)$ for any $n$ and $0 \leq t \leq T$. Since $T>0$ is arbitrary, uniqueness follows.

5. Conclusion. In the present paper we have proven global existence of solutions of a diatomic lattice with intersite interactions. The proposed proof has three features which are to be emphasized here. First, by analogy with the monoatomic lattice, the consideration has been restricted only to the positive nonlinear force constants $\left(\gamma_{1}, \gamma_{2}>\right.$ 0 ). Second, we had to impose the condition of equal nonlinear force constants $\left(\gamma_{1}=\gamma_{2}=\right.$ $\gamma$ ). As is clear, in order to describe the most general and physically relevant case, which admits any signs and relations among the force constants, the proof would have to be modified. Finally, the proposed proof does not allow direct transition to a monoatomic lattice, i.e. to the case $m_{1} \rightarrow m_{2}$ and $\kappa_{1} \rightarrow \kappa_{2}$ simultaneously, which has been considered in [4. In this sense the present paper can be considered as an extension of our previous paper.

In the case of a monoatomic lattice, reported in [4, we outlined the generalizations of the theory to other physically relevant types of the nonlinearities such as, for example, the case of the so-called on-site nonlinearity. In the case of a diatomic lattice the mentioned nonlinearity is described by the potential energy of the form $\Sigma\left[\gamma_{1} u_{1}^{p+1}(n, t)+\gamma_{2} u_{2}^{p+1}(n, t)\right]$. The respective generalizations of the theory to that case (as well as to the cases of periodic nonlinear on-site and inter-site interactions) is not as straightforward as in the case of a monoatomic lattice. This situation would require further analysis. 
Acknowledgments. The main support for this research came from the bilateral program Portugal/Brasil via ICCTI/CAPES being developed at the Federal University of Rio de Janeiro and the University of Lisbon. The first author was partially supported by a European grant from COSYC, no. HPRN-CT-2000-00158.

\section{REFERENCES}

[1] Abdullaev, F. Kh., Darmanyan, S.A. and Garnier J., Modulational instability of electromagnetic waves in inhomogeneous and in discrete media. In Progress in Optics, Edit. E. Wolf, 44, 303-366 (2002).

[2] Bona, J.L. and Saut, J.L., "Dispersive blowup of solutions of generalized Korteweg-de Vries equations". J. Diff. Equations 103, 1993, 3-57. MR1218737 (94h:35225)

[3] Konotop, V.V., "Small-amplitude envelope solitons in nonlinear lattices". Phys. Rev. E 53, 2843 (1996). MR1388219 (97a:82063)

[4] Perla Menzala, G. and Konotop, V.V., "On global existence of localized solutions of some nonlinear lattices", Applicable Analysis, 75, 157-173 (2000). MR1784817 (2001e:37102)

[5] Perla Menzala, G. and Konotop, V.V., "Uniform decay rates of solutions of some nonlinear lattices", Nonlinear Analysis, 54, 2003, 261-278. MR 1979733 (2004c:37200)

[6] Scott, A.C., Nonlinear Science, Oxford University Press (1999). MR1718250 (2001h:37002)

[7] Sulem, C. and Sulem, P., Nonlinear Schrödinger equation: Self-focusing and wave collapse, SpringerVerlag, Berlin (1999). MF1696311 (2000f:35139)

[8] Ziman, J.M., Principles of the Theory of Solids (Cambridge University Press, 1964). MR0165927 $(29: 3207)$ 